\title{
Simulation Studies on the Performance of BCI
}

\author{
Kasa Lalith Kumar1), Swathi Kalam²)
}

\begin{abstract}
A large amount of persons approximately the world knowledge the unwell belongings of beating of action, rendering them reliant on others to perform even the most essential errands. In any case, that could change, due to the most recent accomplishments in the Brain-Computer Interface (BCI), which could enable them to recapture a segment of their lost freedom. Indeed, even ordinary people may likewise have the capacity to use Brain Chip Technology to improve their association with the advanced world-if they will get the embed. The term 'Mind Computer Interface' alludes to the immediate connection between a solid cerebrum and a PC. Serious endeavors and research in this BCI field over the previous decade have as of late brought about a human $\mathrm{BCI}$ implantation, which is incredible news for every one of us, particularly for the individuals who have been surrendered to spending their lives in wheel seats. This Brain Chip Technology is a stage for the advancement of an extensive variety of other helping gadgets. This paper concentrates on the Brain Chip Technology which encourages quadriplegic individuals to do things like checking email, turning the TV, lights on or off with simply their contemplations. Likewise the meaning of Brain-Computer Interface, the essential objective of planning Brain entryway, the fundamental components of Brain Gate, the exploration work led on it at various Universities and some inadequacies of Brain Gate were additionally introduced.
\end{abstract}

Keywords: Genetic Algorithm, Genetic based Function System, Cryptography, Encryption, Decryption.

\section{Introduction}

An implantable mind PC interface the measure of a headache medicine has been clinically tried on people by American organization Cyber energy. The 'Mind Gate' widget will provide incapacitated or engine debilitated patients a method of communication throughout the explanation of consideration into coordinate PC organizes. A Brain-Computer Interface in some cases called a straight neural edge or a mind machine interface (BMI) acknowledges orders straightforwardly from the human or creature cerebrum without requiring physical development and can be utilized to work a PC or different innovations. This wide term can depict numerous genuine and hypothetical interfaces. In this meaning

Received(November 1, 2017), Review Result(1st: November 29, 2017, 2nd: December 31, 2017), Accepted(January 5, 2018)

1) (Professor) Department of Computer Science and Engineering Vignan's Institute of Information Technology Visakhapatnam, AP

2) (Professor, Corresponding Author) Department of Computer Science and Engineering Vignan's Institute of Information Technology Visakhapatnam, AP

email: swathi.kalam@gmail.com 
of Brain-Computer Interface the word cerebrum mean the cerebrum or sensory system of a natural life shape as opposed to the psyche. PC implies any handling or computational gadget shape a coordinated circuit to silicon chip.

The term 'Cerebrum Computer Interface' alludes to the immediate communication between a sound mind and a PC. The objective of the Brain Gate program is to build up a quick, dependable and unpretentious association between the mind of an extremely debilitated individual and a PC. The point of planning this chip is to furnish deadened people with a portal through which they can get to the wide abilities of PCs, control gadgets in the encompassing condition, and even move their own particular appendages. Specialists at the University of Pittsburgh have officially shown that a monkey can encourage itself with an automated arm essentially by utilizing signals from its cerebrum, a propel that could improve prosthetics for individuals, particularly those with spinal string wounds. The term 'Brain-Computer Interface' alludes to the immediate cooperation between a solid mind and a PC. The objective of the Brain Gate program is to build up a quick, dependable and unpretentious association between the mind of an extremely impaired individual and a PC. The point of outlining this chip is to give deadened people a portal through which they can get to the expansive capacities of PCs, control gadgets in the encompassing condition, and even move their own particular appendages. Scientists at the University of Pittsburgh have officially shown that a monkey can bolster itself with a mechanical arm just by utilizing signals from its cerebrum, a propel that could improve prosthetics for individuals, particularly those with spinal string wounds.

Presently, utilizing the Brain Gate framework in the present human trials, a 25 year old quadriplegic has effectively possessed the capacity to switch on lights, modify the volume on a TV, change channels and read email utilizing just his cerebrum. Vitally the patient could do these undertakings while carrying on a discussion and moving his head in the meantime. John Donoghue, the executive of the Department of Neuroscience at Brown University, drove the first research venture and went ahead to help establish Cyber energy, where he is right now boss logical officer administering the clinical trial. It is normal that individuals utilizing the Brain Gate framework will utilize a PC as the portal to scope of self-coordinated exercises. These exercises may stretch out past run of the mill PC capacities (e.g., correspondence) to incorporate the control of articles in the earth, for example, a phone, a TV and lights.

Typically the cerebrum is associated with an outside PC framework through a chip made out of terminals. Presently it is conceivable to embed this chip into the mind's engine cortex (the piece of the cerebrum that controls the developments of the appendages). This enables us to record the electrical action of neurons terminating and utilize PCs to change over the signs into activities by applying signal handling (calculations utilized for the preparing, enhancement and understanding of 
signs). Extraordinary endeavors and research in this field over the previous decade have as of late brought about a human BCI implantation, which is awesome news for every one of us particularly for the individuals who have been surrendered to spending their lives in wheel seat.

\section{Literature Review}

Wolpaw depicts recognizing subjects' mu-disposition adequacy, portrayed as the square-establishment of the spooky EEG control at $9 \mathrm{~Hz}$, using two scalp-mounted anodes arranged close zone $\mathrm{C} 3$ in the International 10/20 System and a propelled signal dealing with board dismembering steady EEG in $333 \mathrm{~ms}$ sections, and using it to drive a cursor up or down on a screen toward a target set discretionarily at the best or base. An examination director preset the traverse of the degrees and number of cursor improvement steps designated to each range for each subject in the midst of testing going before each trial. Degrees were set so that the commonest mu-rhythm amplitudes $\quad<4$ microvolt's) left the cursor set up or moved it downwards sensibly while higher amplitudes $(>4$ microvolt's) moved it upwards in growing bobs. Weights were adjusted as subjects indicated better control of their mu-inclination amplitudes for all finished concentrations in repeated trials. Wolpaw substantiates subjects' insightful think control over mu-rhythm sufficiency in three courses: by performing repeat examination up to $192 \mathrm{~Hz}$ on subjects in the midst of cursor improvement trials and fail to find any association between mu-Rhythm changes and the higher frequencies related with strong (EMG) activity; by subjects' declarations about not making contra sidelong advancements and observing none; and by fail to find any association between mu-musicality changes and back scalp records of the visual alpha-disposition.

Four out of five subjects increased astounding control over their mu-musicality plentifulness in the midst of 12 45-minute sessions over a period of two months. Correctness's of $80-95 \%$ target hits across finished guineas pigs were expert and rates of 10-29 hits for each minute. Separated examination of two subjects' unrefined EEG data (see underneath) if incredible help for Wolpaw's exploratory results. McFarland used fundamentally the same exploratory setup and displayed more vital exactness necessities on four subjects' undertakings to position a cursor by strategies for mu-temperament control. Examination of the ordinary division between the point of convergence of the goal and the cursor in the midst of succeeding trials demonstrated that all subjects diminished the partition and three out of four inside and out. 


\section{Architecture Model of the System}

Let us initially talk about the three sorts of $\mathrm{BCI}$ before affecting the genuine ramifications of $\mathrm{BCI}$ and its purposes. These sorts are chosen the premise of the strategy utilized for the interface. Every methods has a few focal points and in addition a few hindrances.

\section{Investigational Brain Computer Interface Software for the Modular EEG (The ABI software):}

ABI is the software used or the software available in the market for the Modular EEG which can be used to implement various applications and also for the Brain Computer Interface (BCI). at the present time, the research on the area of $\mathrm{BCI}$ is very much high in the field of research and also the available of resources for this sort of technology by general users is very much high and tight and also the resource is very much useful for the latest research applicant areas. The usage of the current research is very much high in the market is very high and also the very much tight or difficult for implementing the research in the recent current areas. The current software is highly useful for various users like the current day users and also for the general users who can use the current software for their general applications and also for the simple day to day applications.

By and large, taking into account a perceived occasion with mind harming potential (that isn't probably going to be credited to any occasions in the intrauterine condition) in a formerly well newborn child[1]. Regular reasons for adolescence ABI incorporate awful cerebrum damage (TBI), stroke, disease, cerebral tumor and encephalitis[2]. Late advances in medicinal reaction have prompted diminished death rates following mind damage (counting TBI, cerebrum tumor and stroke), with more people living with the sequelae of $\mathrm{ABI}[3]$. More than 600,000 Australians have cerebrum damage with Queensland having the most elevated predominance $(2.5 \%$ contrasted with $1.8 \%$ for whatever is left of Australia)[2]. Upwards of two out of three of these individuals obtained their mind damage before 25 years old with the larger part supporting a TBI[4]. The evaluated lifetime cost per occurrence case is AUD\$ 4.8 million for direct and serious TBI of which $19 \%$ is borne straightforwardly by state governments[3]. These figures incorporate money related costs required with human services, hardware and alterations, long haul mind, efficiency misfortunes and weight of sickness costs. This features the significance of creating powerful medicines and intercessions past intense therapeutic care to improve post-damage results.

Numerous youngsters with an ABI encounter steady scholastic, word related, physical, enthusiastic 
behavioral and intellectual disabilities prompting diminished personal satisfaction (QOL)[5-7]. Behavioral shortages, for example, uneasiness and wretchedness may likewise fall apart over the long run as a result of harm to particular regions of the mind and expanded animosity and hyperactivity may not show until quite a while after damage[4][6]. These debilitations affect on a youngster's capacity to effectively take an interest in restorative intercessions post release.

Albeit engine results recuperate more rapidly than psychological aptitudes after ABI, continuous physical hindrances may remain. Physical hindrance is firmly identified with mind damage seriousness, whereby more noteworthy cerebrum damage seriousness predicts poorer physical (and subjective) results[5-7]. Regular physical impedances following $\mathrm{ABI}$ incorporate changes in muscle tone, weakened adjust and sensation, diminished quality and coordination[1][2]. Aging and Careers found that about the majority of the 20,000 youngsters (90\%) who were recognized as having an $\mathrm{ABI}$ as a fundamental or related analysis were accounted for to have "extreme or significant center movement restrictions"[4]. Engine troubles affect a tyke's capacity to take part in exercises of day by day living (ADL, for example, getting dressed and strolling. Recuperation of useful capacities and support for continuous securing of formative aptitudes was the main objective of patients who were joining and the patients who were leaving the hospital from recovery also the youngsters can come back to taking an interest in age fitting exercises.

Psychological weakness is a typical outcome of $\mathrm{ABI}[2][4][5]$, with youngsters encountering challenges with memory, consideration, fixation, official brokenness and a potential decrease in scholarly capacity after some time[4][5]. Past examinations have detailed that it is frequently official control over psychological procedures, as opposed to particular procedures themselves (e.g. memory) that is hindered and requires restoration[1][2]. Regularly such psychological deficiencies following an $\mathrm{ABI}$ can prompt poor school accomplishment, behavioral issues and effect individual relations and business openings[3].

\section{Simulation and recording}

In the current simulated model or the current simulator we are considering for the current utilization and also for the implementation will have two modes for the operation. The two modes can be used for the simple two sets of trials. The current simulator is used for the studies on the performance of the BCI. It is used further for the performance of the brain interface and also for the analysis of the brain recording for the brain data. 


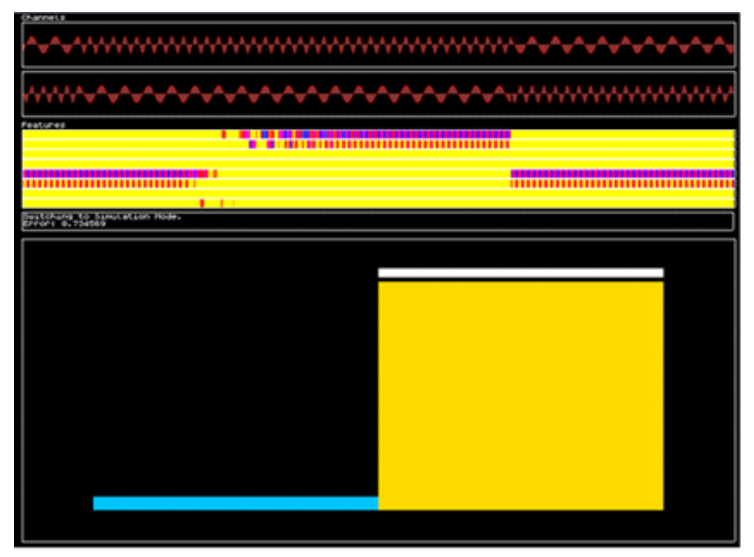

[Fig. 1] ABI Software Screenshot for the Initial Values

\subsection{Training}

The preparation set utilized for this design is the arrangement of the last Trial Buffer recorded trials' highlights. Illustration: Suppose you have recorded 450 trials, and Trial Buffer $=250$. At that point the framework removes the highlights of the 160 last recorded trials to shape the preparation set. Preparing time relies on the many-sided quality of the preparation information and the measure of recorded information. The preparation information isn't generally detachable. On the off chance that the mental assignment for class 1 is excessively comparable, making it impossible to the mental errand for class 2, at that point the neural net won't have the capacity to do the division: this isn't enchantment.

[Table 1] Training Data

\begin{tabular}{|l|l|l|l||}
\hline \multicolumn{1}{|c|}{ Substate } & \multicolumn{1}{|c|}{ Duration } & \multicolumn{1}{c|}{ Description } & Screenshot \\
\hline Preparation & $\begin{array}{l}\text { T preparation } \\
\text { seconds }\end{array}$ & $\begin{array}{l}\text { The BCI does not } \\
\text { display anything but } \\
\text { the EEG data and the } \\
\text { features. The user } \\
\text { can relax during this } \\
\text { time. }\end{array}$ & | \\
\hline Prerecording & $\begin{array}{l}\text { T prerecording } \\
\text { seconds }\end{array}$ & $\begin{array}{l}\text { The BCI displays the } \\
\text { class target by } \\
\text { indicating a white } \\
\text { target line. The user } \\
\text { should start to } \\
\text { perform the essential } \\
\text { task associated to the } \\
\text { target class. }\end{array}$ & \\
\hline Recording & Srial Length & $\begin{array}{l}\text { The BCI displays the } \\
\text { bars indicating } \\
\text { which classes are } \\
\text { identified in each } \\
\text { time sequence. }\end{array}$ & \\
\hline
\end{tabular}


Pressing the F3 key, the scheme begin to teach the neural net data that was available.

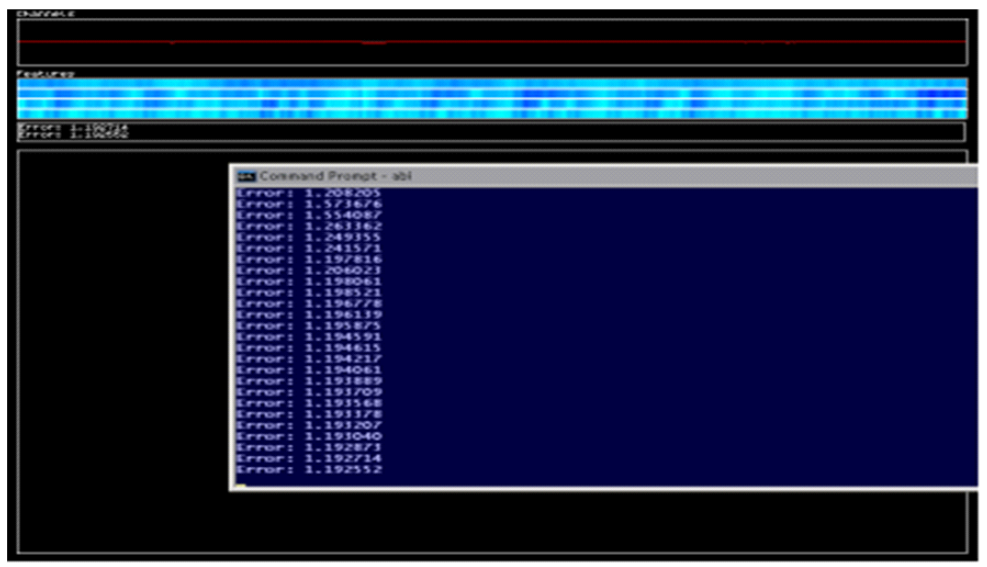

[Fig. 2] The Scheme Begin to Teach the Neural Net Data That was Available

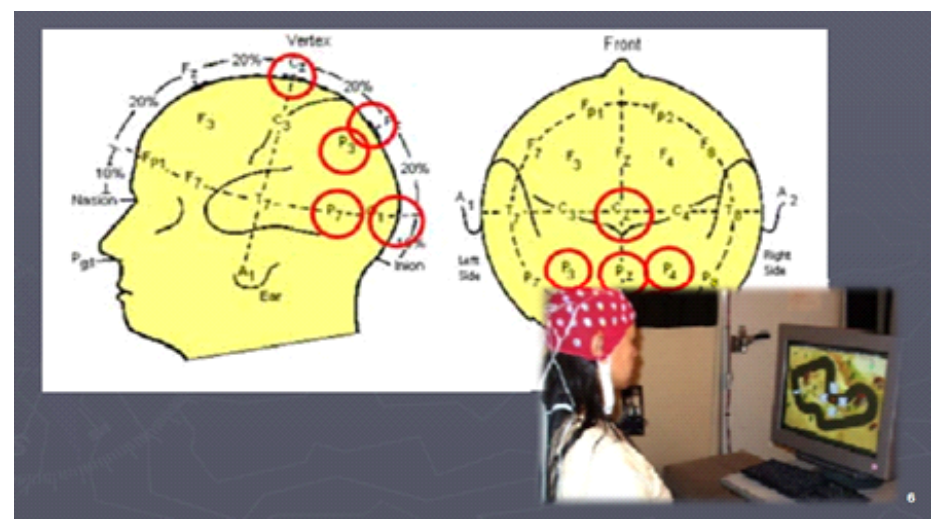

[Fig. 3] Electrode Positions

While working with the current problem, the EEG information evidence collected so far is spared into the document. You can open a trial document with a straightforward content tool and perceive how the trial information has been recorded. Just crude EEG information and the class name is recorded: the highlights, which relate to the genuine preparing information, are registered on-the-fly. On the off chance that you begin $\mathrm{ABI}$, it will stack the trial file indicated in the arrangement record on the off chance that it exists, or make another one if not. On the off chance that the trial file doesn't coordinate the design document's details, at that point $\mathrm{ABI}$ prematurely ends its execution. So you must be mindful so as to utilize rectify trial files and setup documents. EEG recording between various executions of the $\mathrm{ABI}$ framework is annexed to the trial chronicle. This enables you to 
fabricate your preparation set in various sessions. Be mindful so as to utilize similar anode settings. Some have announced that the acknowledgment rate drops between various sessions.

\section{Conclusions}

The likelihood that EEG movement or other electro-physiological measures of cerebrum capacity may give new non-strong channels to correspondence and control (i.e., BCls) has been a subject of hypothesis for a long time. In the course of recent years, various gainful BCI innovative work programs have been started. These undertakings concentrate on growing new augmentative correspondence and control innovation for those with extreme neuromuscular issue, for example, ALS, cerebrum stem stroke, and spinal string damage. In this way, the client and the BCI framework need to adjust to each other both at first and consistently to guarantee stable execution. This reliance on the shared adjustment of client to framework and framework to client is the major guideline of BCI operation. BCI innovative work is a characteristically interdisciplinary issue, including neurobiology, brain research, building, arithmetic, software engineering, and clinical restoration. Its future advance and inevitable useful effect rely upon various basic issues. The relative favorable circumstances and disservices of non-obtrusive and intrusive strategies stay to be resolved. From one perspective, the full limits of non-obtrusive techniques are not clear; then again, the long haul wellbeing and dependability of intrusive strategies are questionable.

\section{References}

[1] D. M. Taylor, S. I. Tillery, and A. B. Schwartz, Direct cortical control of 3D neuroprosthetic devices, Science, (2002), Vol.296, pp.1829-1832.

[2] J. Wessberg, C. R. Stambaugh, J. D. Kralik, P. D.Beck, M. Laubach, J. K. Chapin, J. Kim, S . J.Biggs, M. A. Srinivasan, and M. A. Nicolelis, Real-time prediction of hand trajectory by ensembles of cortical neurons in primates, Nature, (2000), Vol.408, pp.361-365.

[3] M. D. Sermya, N. G. Hatsopoulos, L. Paninski, M.R. Fellows, and J. P. Donoghue, Instant neuralcontrol of a movement signal, Nature, (2002), Vol.416, pp.141-142.

[4] J. K. Chapin, K. A. Moxon, R. S. Markowitz, and M. A. Nicolelis, Real-time control of a robot armusing simultaneously recorded neurons in the motorcortex, Nature Neuroscience, (1999), Vol.2, pp.664-670.

[5] J. R. Wolpaw, N. Birbaumer, D. J. McFarland, G.Pfurtscheller, and T. M. Vaughan, Brain-computer interfaces for communication and control, Clinical Neurophysiology : Official Journal of the International Federation of Clinical Neurophysiol-408, (2002), Vol.113, pp.361-5, pp.141-2, pp.767-91. 